\title{
Gauging the capacity for concurrent motor planning in human fMRI with information constraints in bounded rational decision-making models
}

\author{
Sonja Schach ${ }^{1, *}$, Axel Lindner ${ }^{2,3}$, Daniel A. Braun ${ }^{1}$
}

\begin{abstract}
While traditional theories of sensorimotor processing have often assumed a serial decision-making pipeline, more recent approaches have suggested that multiple actions may be planned concurrently and vie for execution. As most of the evidence for the latter comes from monkeys, here we study concurrent prospective motor planning in humans by recording functional magnetic resonance imaging (fMRI) during a sequence planning task with multiple potential targets. We find that fMRI activity in premotor and parietal brain areas modulates both with the sequence complexity and the number of targets. We test the hypothesis that fMRI activity is best explained by concurrent planning as opposed to the incomplete determination of a single action plan. We devise a bounded rationality model with information constraints that optimally assigns information resources for planning and memory for this task and determine predicted information profiles according to the two hypotheses. When regressing fMRI activity using this model, we find that the concurrent planning strategy provides a significantly better explanation of the fMRI modulation profile. Moreover, we find that concurrent planning is limited for most subjects, as expressed by the best fitting information capacities. We conclude that bounded rational decision-making models allow relating both behavior and neural representations to utilitarian task descriptions based on bounded optimal information-processing assumptions.
\end{abstract}

keywords: information capacity, human motor planning, fMRI, prospective planning, bounded rationality model

\section{Introduction}

Traditional theories of sensorimotor processing often consider a sequential pipeline from perception to action, where in between the cognitive system makes a decision that is subsequently implemented by the motor system that plans and executes the corresponding action $[1,2,3,4,5,6]$. In this view perceptual, central, and motor representations do not overlap and are separated in time. Thus, it is often assumed that the value of different alternatives is computed and used for decision-making in prefrontal regions,

* corresponding author. sonja.schach@uni-ulm.de

1 Insitute of Neural Information Processing, University of Ulm, Ulm, Germany

2 Department of Psychiatry and Psychotherapy, University of Tübingen, Tübingen, Germany

3 Centre of Neurology, Division of Neuropsychology, Hertie-Institute for Clinical Brain Research, University of Tübingen, Tübingen, Germany 
which is then subsequently translated into action planning in premotor regions, including parietal, precentral, and subcortical regions [1]. In contrast, more recent frameworks, like the affordance competition hypothesis $[7,8]$, have put forward the notion that actions might be planned concurrently in several frontoparietal brain areas and vie for execution, whenever opportunities for multiple potential actions arise at any one time.

Neurophysiological studies in monkeys have found increasing evidence, that multiple potential actions are planned in parallel and competitive potential plans are simultaneously represented in posterior parietal cortex (PPC) [9], including superior parietal lobule (SPL) and anterior intraparietal sulcus (antIPS), and dorsal premotor cortex (PMd) $[10,8]$. Moreover, it has been suggested that neurons that are involved in motor planning are often also involved in the decision-making process $[11,8]$. In how far particular decisions are accompanied by more integrated or more serial processing may also depend on the task specification, as shown by Cui et al. [2] for spatial and non-spatial decisions. In particular, their results show that different areas in the posterior parietal cortex encode both potential and selected reach plans (parietal reach region) or encode only selected reach plans (parietal dorsal area), suggesting a parallel visuomotor cortical circuitry for spatial effector decisions. Some studies have even suggested that concurrent planning can take place after movement onset in a way that is effecting movement kinematics and variability during reaching $[12,13,14]$.

Although there is ample evidence for neural correlates of prospective planning in humans in parietal and premotor planning-related areas such as SPL and PMd [15], so far most evidence for parallel prospective planning has been acquired in monkey studies. Here we ask whether 1) parallel prospective motor planning also happens in humans and 2 ) if there is a certain capacity limit for concurrent planning and how this could be quantified. Prospective parallel planning could bring an essential benefit in the preparation for multiple potential futures by enabling to react more quickly to the upcoming scenario, though, it would be the more cost-intensive strategy compared to serial planning.

What makes parallel prospective planning so expensive is that it does not only require to narrow down the number of action possibilities with incoming sensory evidence $[16,17]$, but also to proactively predict possible future world states by sampling from an internal model and preparing multiple action plans from which one finally gets selected $[18,7,19$, 20]. It is immediately clear that parallel planning cannot be applied indefinitely and that there must be bounds on the amount of information processing: this could be the amount of plans or the precision and granularity of the plans or the probability that particular plans are instantiated.

Similar to previous studies that have quantified the capacity of working memory using information theory to measure how well different items can be told apart [21, 22, 23, 24, $25,26,27,28,29]$, we use information theory to quantify the planning capacity in the context of a sensorimotor task defined by a task utility. This can be achieved by designing optimal choice models with information constraints resulting in an abstract model of bounded rational decision-making where resources are measured in units of information with the understanding that other resource measures like time or energy consumption can ultimately be converted into limits in information. In previous studies [30,31], we have investigated such optimality models with information bounds in the context of motor planning by manipulating both permissible reaction times and prior distributions over possible world states. In these behavioural experiments reaction time was used as a proxy for information cost underlying any process of uncertainty reduction.

Here we use this approach for the first time to measure how much of the variability of brain signals can be accounted for by bounded optimal information flow. To this end, 
we correlated the magnitude of the fMRI BOLD signal during the planning phase of a motor task with theoretical information-processing costs predicted by an optimal choice model with information constraints. In particular, we compared two versions of evaluating the model designed with respect to the ongoing debate of serial and parallel processing in prefrontal, premotor and parietal brain areas. According to the serial hypothesis, parameters for different action possibilities are not determined until enough evidence has accumulated for a decision, whereas the parallel processing hypothesis assumes that multiple actions are specified simultaneously so that their information processing costs have to be added. We used the model predictions to test the hypothesis $H 1$ that the motor system devises multiple concurrent motor plans by anticipating proactively possible future world states against the null hypothesis $\mathrm{HO}$ that uncertainty in the action space is simply reduced given the current sensory evidence without prospective planning for possible anticipated futures (see Figure $1 \mathrm{~A}$ ). Finally, we can used the model to gauge the information capacity for parallel planning and find that concurrent planning is limited, in particular in complex task conditions.

In line with previous studies, we found related brain activity in the parietal-frontal planning system (SPL and antIPS and premotor areas PMd) and further areas related to movement planning (see also Supplementary Figure S1), and our correlation measures suggest that this activity modulation is consistent with a bounded optimal information flow implementing parallel prospective motor planning.

\section{Results}

\section{Behavioral performance analysis}

In a delayed-response-task (DRT), subjects were shown a cue stimulus with possible targets that were highlighted across four concentrically ordered response panels. They were verbally encouraged during the delay phase to plan movement sequences to all potential targets, and to execute one of them, when shown the appropriate selection stimulus during the response phase (see Figure 1). Movement sequences could vary in length (2-step, 3 -step and 4-step condition) and consisted of multiple directional button clicks to move a cursor on a screen. To enforce engagement during the delay phase, the selection stimulus did not indicate the actual selected target directly, but only indicated the correct panel half containing the selected target, such that subjects could identify the selected target uniquely only if they remembered the information contained in the initial cue stimulus. In total, our task design contrasted 12 DRT conditions, that did not only vary in the movement sequence length, but also in the number of possible targets (1,2 or 4), where only the 2-target case allowed an additional condition ' 11 ' with two targets in the same panel, and all other conditions had only one possible target per panel. Consequently, the 12 DRT conditions were distinguished by different cognitive load on planning due to multiplicity of potential targets and sequence length.

We assessed subjects' behavioral performance in terms of reaction times, movement times and error rates and compared to a control condition (CT), where the cue stimulus did not contain any information about the target location and therefore subjects could not plan during delay.

We found that reaction times increased with the number of potential targets $(p<$ 0.001, rmANOVA linear within-subject effect), suggesting that subjects engaged in more complex planning as the number of potential targets increased (see Methods section for details on the statistical analysis). In contrast, movement path length had no significant 


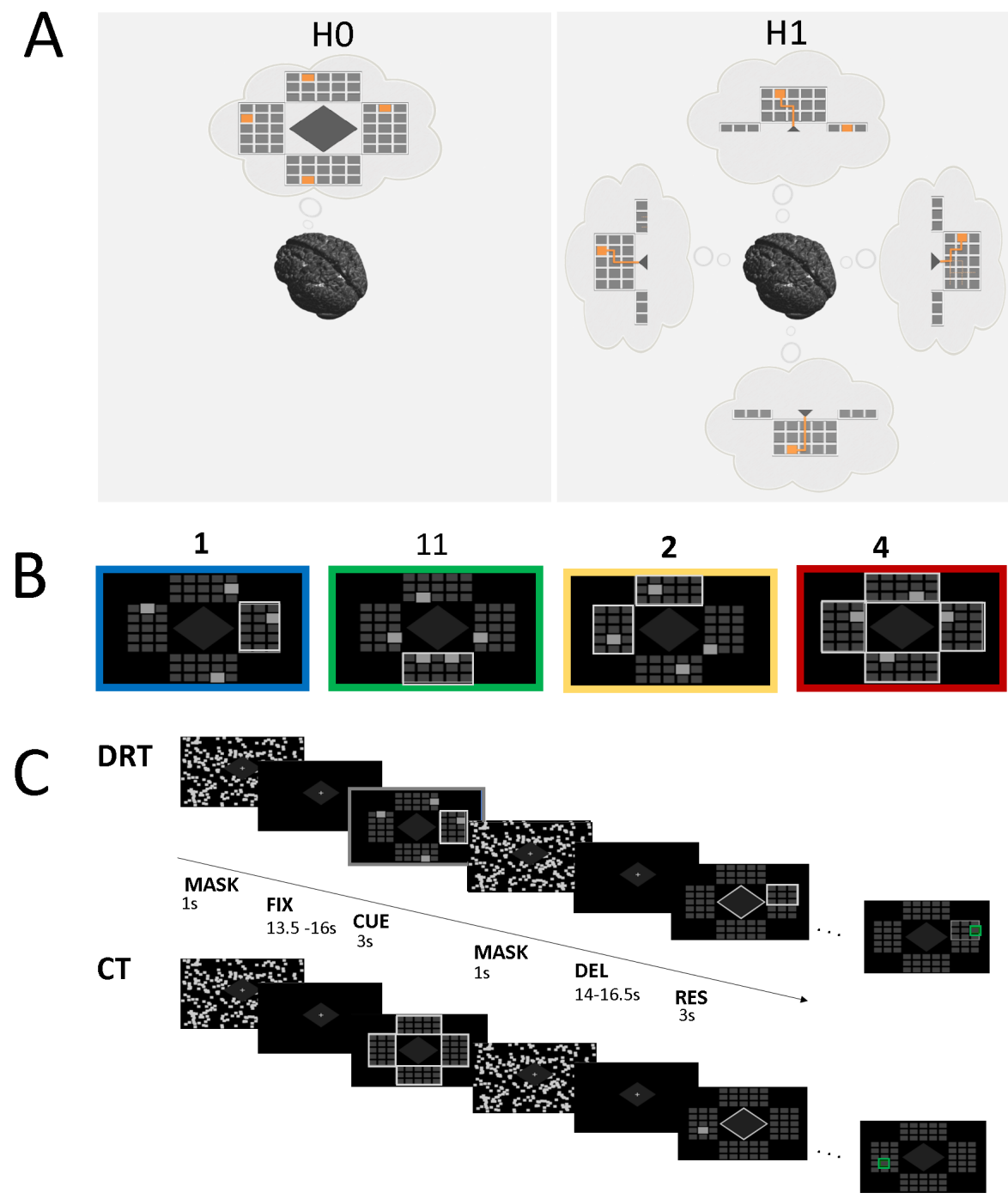

Figure 1: (A) Model Hypothesis. We correlated theoretical memory and planning information with measured fMRI activity in relevant brain areas and tested two hypothesis: $\mathrm{H} 0$, where information costs correspond to uncertainty reduction and H1, where on top of the uncertainty reduction information costs for prospective planning are required, because possible future events are anticipated in the delay phase. (B) The four target planning conditions differed in the number of possible target locations. Subjects could see target positions highlighted. Potential targets to consider for planning were indicated by a frame around the field areas encompassing the relevant targets. Subjects were instructed to plan a single movement sequence towards one target ('1'), two similar ('11') or two differing movements ('2') or four movements ('4') respectively and execute the planned movement in the following phase, after the solution was indicated. (C) Trials of the motor planning conditions in the delayed response task (DRT) and control condition (CT) were randomized and had a similar timeline. Each trial started with a fixation period (FIX) of random duration, a cue phase (CUE) followed by a visual mask (MASK), a delay phase (DEL) of random duration for planning and a response phase (RES), where subjects should execute the previously planned movement to the instructed target. In the control task, no target cues were presented during the cue phase and therefore no goal-directed actions could be planned during the delay phase. In the response phase of the CT subjects were finally informed about the target location and had to move without previous planning. 
effect on reaction times, even though the different sequence lengths had a clear impact on movement times as shown in Figure $2 \mathrm{~B}$ ( $p<0.0001$, rmANOVA factor steps). Compared to the control condition $\mathrm{CT}$ that did not allow for any planning during delay, reaction times in the easy planning conditions (' 1 ' and '11') were significantly reduced before movement execution ( $p<0.0001$ and $p=0.004$, rmANOVA factor condition), whereas for the two more complex planning conditions ' 2 ' and ' 4 ' such a reaction time advantage was not measurable (see Figure $2 \mathrm{~A}$ ). In the control condition, the target was explicitly shown after the delay and movements could be planned without the need for memorization, which results in the lowest error rates in this condition (see Figure $2 \mathrm{C}$ ). In contrast, the DRT conditions required some form of memorization of the cue stimulus, which allowed for additional errors due to memory failure, which consequently led to higher error rates in the more complex DRT conditions ( $p=0.001$, rmANOVA, condition ' 4 ' vs CT). Memorization during cue presentation was also accompanied by varying amounts of eye movements, but crucially during the delay phase the number of saccades per second did not significantly differ between the different planning conditions and control conditions (see Figure $2 \mathrm{D}$ ). This absence of difference in eye movements during the delay phase will be important in the next section, where we study fMRI activity during the planning delay period and need to exclude additional effects on fMRI from eye movements.

\section{fMRI analysis}

The relative changes of the fMRI signal due to planning during the delay phase compared to a pre-stimulus baseline can be seen for the different conditions in Figure $3 \mathrm{~A}$ for the exemplary planning area SPLI. As expected, the activity was lowest during the control condition CT that does not provide any information for planning. The activity increased with the complexity of the planning problem with the highest activity for the target condition '4' with 4 steps. As a control measure, primary visual area V1l that is thought not to be involved with motor planning showed no such modulation (see Figure $3 \mathrm{~B}$ ). Due to the slow BOLD haemodynamic response function (HRF) visual processes and eye movement related activity during cue presentation could influence the early delay phase of planning. Differences in pure planning activity for different task conditions are therefore reflected in the late delay phase. However, the early delay phase is still representative for the modulation of the number of steps because the visual cue does not explain related activity differences.

We fitted a general linear model (GLM) to the time series data measured in each voxel and used the regression model to determine regions of interest that show significant functional planning related activity on the group level. To this end, all 12 DRT conditions were contrasted with the control condition $\mathrm{CT}$ in each individual subject within the delay period. The group map in Figure $3 \mathrm{C}$ based on second-level analysis showed significant differences across all subjects in planning-related areas including SPLl/SPLr, PMdl/PMdr, DLPFCr, antIPSl/antIPSr, AICl/AICr, SMA, cer6l/cer6r and cer8r. For each of the predefined ROI areas, we extracted GLM parameter estimates reflecting planning related changes in fMRI BOLD signal amplitudes in different task conditions of varying planning complexity. In our major planning area of interest SPLl, GLM parameters differed significantly between individual planning conditions distinguished by the number of targets $(p<0.0001$, rmANOVA $)$ and number of movement steps $(p=0.001$, rmANOVA $)$ but there was no significant interaction effect between target planning conditions and number of steps. For results of different ROIs please refer to Supplementary Figure S1. 

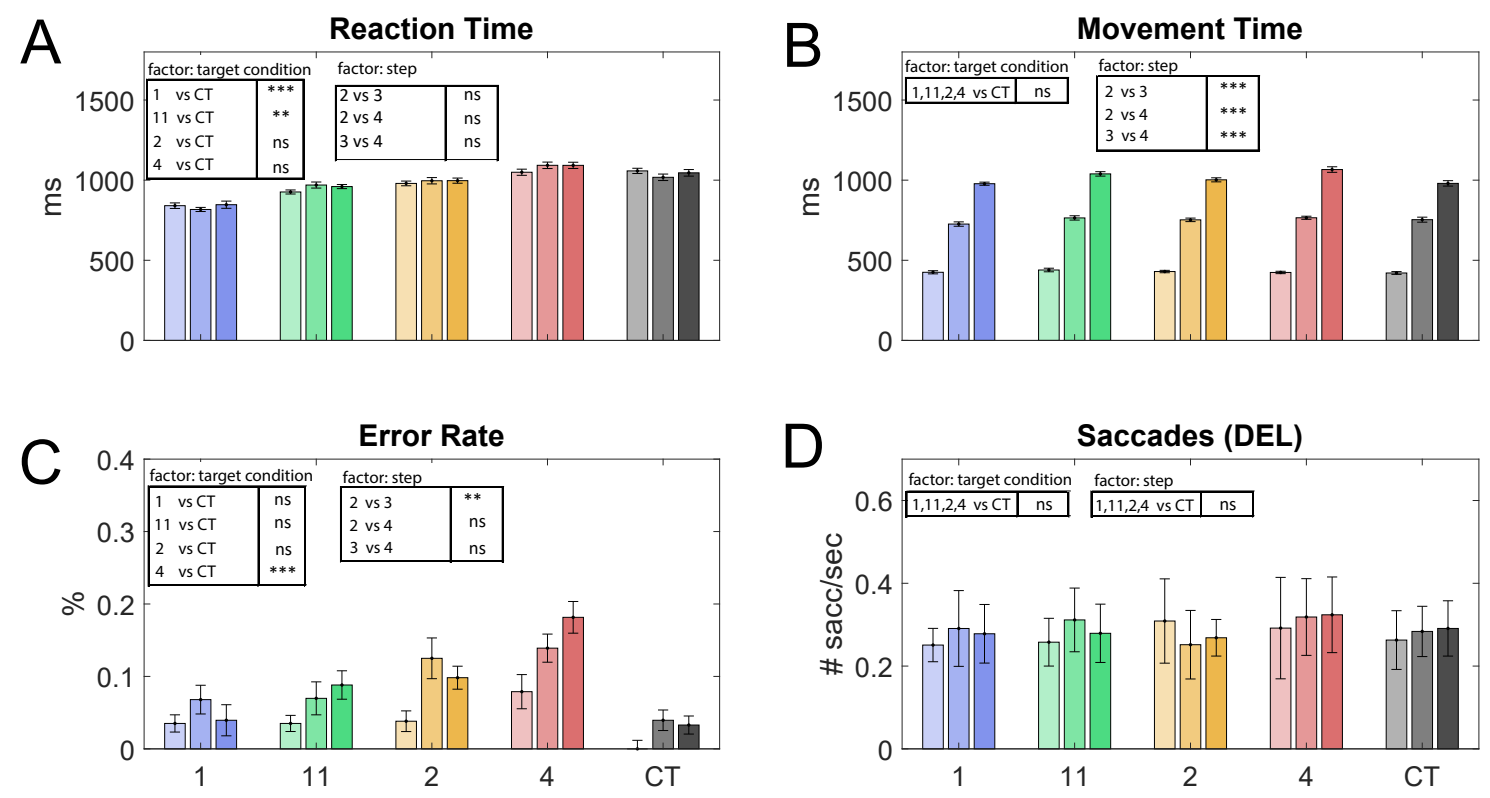

Figure 2: Behavioral performance. Results are reported as the mean over subjects' individual task performance and normalized standard error (according to [32] to eliminate the between-subject variance, which does not contribute to the within-subject effect of the study). Post-hoc pairwise 2-way repeated measures ANOVA results with statistical comparison of factor target condition compared to control (1vsCT, 11vsCT, 2vsCT and $4 \mathrm{vsCT})$ and factor step (2vs3, 2vs4 and 3vs4) are indicated with ${ }^{* * *}$ for $p<=0.001,{ }^{* *}$ for $0.001<p<=0.01$, and ns for non-significant results $(p>0.05)$. (A+B) Reaction times (RT) and movement times (MT) were calculated as normalized averages across individual subjects' means \pm SEM. Reaction times were significantly decreased for easy planning conditions ('1' and '11') compared to control condition CT, which indicates benefit due to planning. RT and MT were higher in the more complex planning conditions ('2' and '4') and did not significantly differ compared to CT. Differences between step-conditions were found for MT but not for RT. (C) Error rates averaged across subjects indicated varying task difficulty over conditions revealed by an increase of target misses for conditions with higher task uncertainty (significant difference for '4' vs CT) and between 2- and 3-stepconditions. (D) During planning delay across subjects there was no significant difference between the average number of eye saccades and therefore saccades cannot explain benefit in reaction times or fMRI activity in planning-related areas. 
A

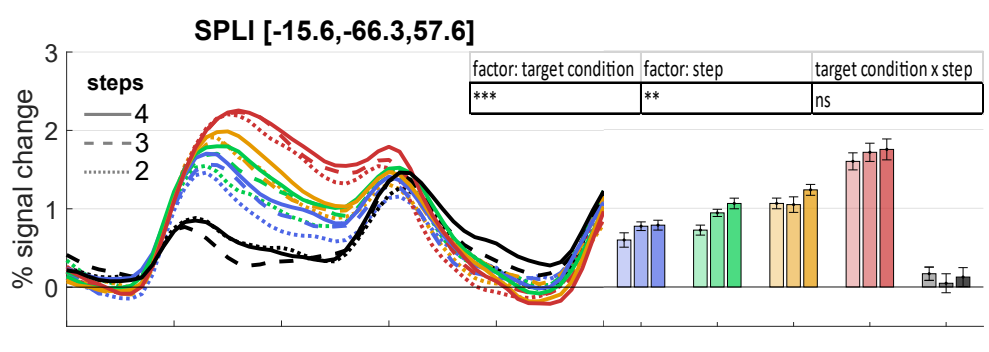

B

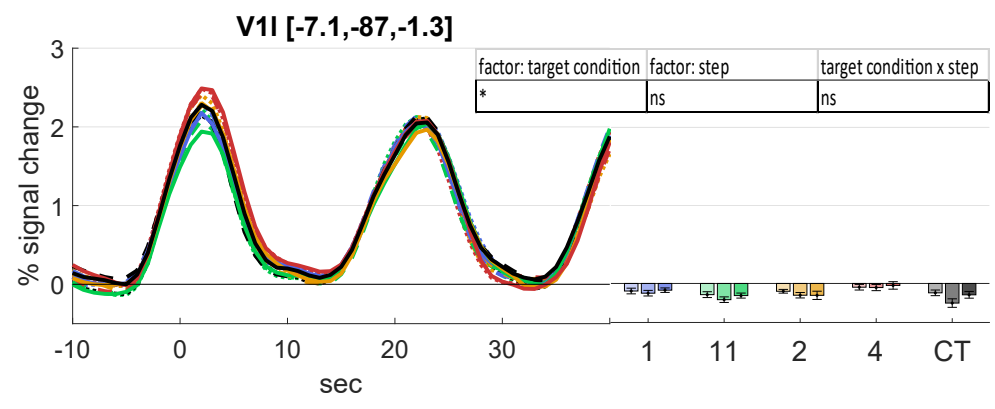

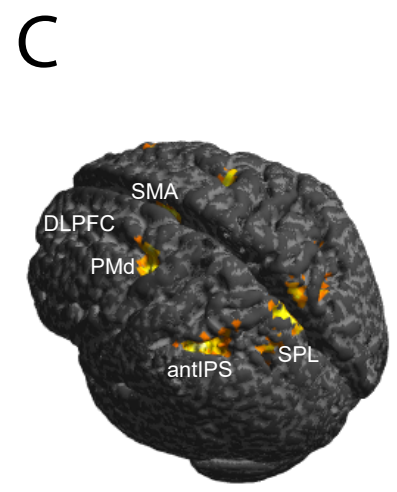

Figure 3: fMRI activity related to motor planning. (A) Time course of raw activity as percentage of signal change normalized to pre-stimulus baseline and activity estimates (beta parameter from GLM) in each planning condition in planning related area SPLl show increasing BOLD amplitude with increasing task complexity. We report across-subjects averages and within-subjects variance as the normalized standard error (according to [32]). Time course activity and GLM estimates of further ROIs are provided in Supplementary Figure S1. Average MNI-coordinates (x, y, z in $\mathrm{mm}$ ) are provided for each ROI. Statistical results are indicated with ${ }^{* * *}$ for $p<=0.001,{ }^{* *}$ for $0.001<p<=0.01,{ }^{*}$ for $0.01<p<$ 0.05 , and ns for non-significant results $(p>0.05)$. (B) In non-planning related area V1l BOLD signal changes were not significantly different between conditions. (C) Second-level activation map shows significant fMRI activity across subjects in planning related areas (SPL, PMd, DLPFC, antIPS, AIC, cer lobus VI and VIII and SMA) during planning delay for statistical contrast of DRT conditions vs. control condition CT ( $p \geq 0.05$ family-wise error FWE-corrected for multiple comparisons). 


\section{Model comparison}

In order to test our hypothesis of prospective concurrent planning against the null hypothesis of uncertainty reduction based on memorization, we compared the fMRI activity modulation to the expected information modulation in an optimal decision-making model with information constraints with and without concurrent prospective planning (hypothesis $\mathrm{H} 0$ and $\mathrm{H} 1$, respectively; see Figure 4). For a detailed description please see the Methods section. Assuming motor planning only in terms of uncertainty reduction due to the perception of the cue stimulus, during the delay phase, the decision-maker updates its internal memory (information reduction $I_{1}$ ) and correspondingly reduces the action uncertainty from a prior over all actions to a posterior over potential actions given the memory (information reduction $I_{2}$ ). We contrasted this null hypothesis $\mathrm{H} 0$ with the assumption of prospective planning, where separate action plans are made for all potential targets. In this alternative hypothesis H1, the memory update is identical to H0, but the information reduction for the action is now composed of the sum of information reductions for each possible motor plan. The exact amounts of information reduction depend on the capacity of the information channels defined by the memory and the action processes. Three example profiles of information modulation with different capacities are shown in Figure 4.

To find out which hypothesis explains our data best, we established (non-negative) multilinear regressions between the fMRI activity modulation and the two information modulations predicted by each of the two hypotheses. For both hypotheses, we first calculated the expected information costs for possible settings of information capacities and we regressed these theoretical information modulations with subject-specific GLM activity estimates over the 12 DRT conditions for all ROIs separately. Figure 5 A shows, exemplarily for brain area SPLI, the $R^{2}$-values of the regressions for the best fitting information capacities for each subject under the two hypotheses (which we refer to as bounded in the Figures 4 and 5). A within-subject comparison of the $R^{2}$-values for the two hypotheses showed that the information profiles under the prospective planning hypothesis H1 provide a significantly better explanation of the fMRI modulation than the null hypothesis $\mathrm{H} 0$ in all planning related ROIs (SPLl: $p=0.0001 \mathrm{rmANOVA}$ ), but not in control area V1l. For further details on the statistical analysis see the Methods section. Moreover, we applied a nested model F-test statistic to both hypotheses to find out whether the predicted planning-specific information reduction $I_{2}$ contributed significantly to the multilinear regression of the fMRI signal. We found that for most of the subjects (15 from 19 for SPLl) under the prospective planning hypothesis H1 the planning information $I_{2}$ improved the regression of the signal modulation significantly (see Figure 5). In contrast, the nested model tests for $\mathrm{H} 0$ revealed that model regressions did not improve significantly by taking $I_{2}$ into account.

To address the question in how far concurrent planning is limited in its capacity, we compared the $R^{2}$-values of the regression with an optimal decision-making model without capacity bounds that we obtain from the bounded decision-making model as a limit case of maximum capacity (which we refer to as not-bounded in Figures 4 and 5). We found that the correlation of measured brain activities in all planning related ROIs was significantly increased for model predictions with subject-individual bounds (SPLl: $p=0.0001$ rmANOVA) compared to an unbounded maximum capacity model. This suggests that judging from the modulation of the fMRI signal alone, it seems that subjects were limited in their information processing. As a control, the $R^{2}$-value of the information modulation in primary visual area V1l was close to zero (see Supplementary Figure S2). The individually fitted information bounds under the two hypotheses $\mathrm{H} 0$ and $\mathrm{H} 1$ can be seen in the 
HO

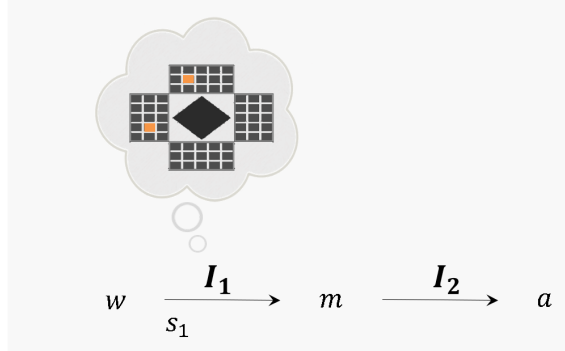

not - bounded

$\underset{\beta_{1}=500, \beta_{2}=500}{\text { not }- \text { bounded }}$

\section{bounded}

$\beta_{1}=4, \beta_{2}=14.5$

$\beta_{1}=40, \beta_{2}=4.5$

:

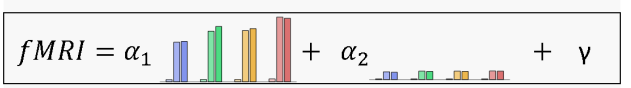

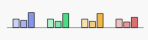

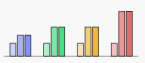

$\mathrm{H} 1$
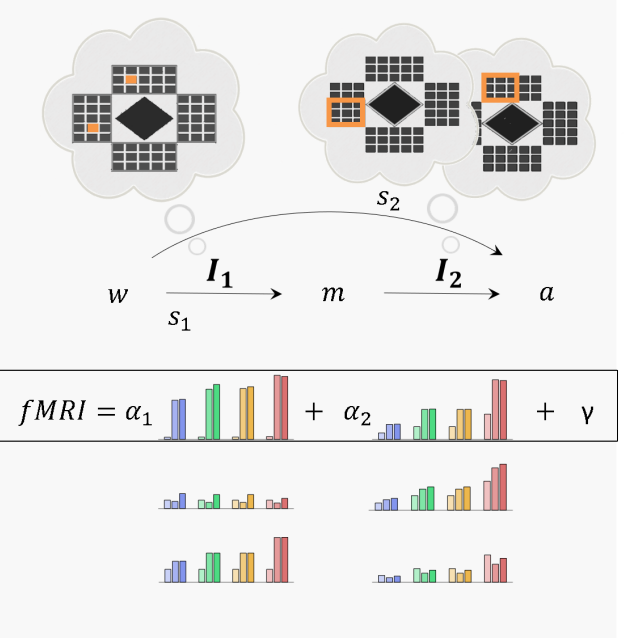

Figure 4: Information theoretic model hypothesis of bounded optimal planning with information constraints. Information about the hidden world state $w \in \mathcal{W}$ (the exact target location) is revealed in two steps. When seeing the combined cue stimulus $s_{1} \in \mathcal{S}_{1}$ indicates all possible target locations, the decision-maker can form a memory $m \in \mathcal{M}$ before perceiving the second stimulus $s_{2}$ that resolves the remaining uncertainty about the actual target $w$ and can finally select an action $a \in \mathcal{A}$ that corresponds to a movement path. Both hypothesis ( $\mathrm{H} 0$ and $\mathrm{H} 1$ ) contrast the predicted information $I_{1}$ and $I_{2}$ in all 12 DRT conditions for a serial and parallel strategy. H0: During delay phase only memory is used to reduce uncertainty about actions. H1: Prospective planning of actions during delay phase requires the anticipation of all possible selection stimuli $s_{2}$ and planning movement sequences in parallel. Theoretical information $I_{1}$ and $I_{2}$ of the bounded rationality model for different memory and planning capacities, defined by model parameters $\beta_{1}$ and $\beta_{2}$, were regressed (using regression coefficients $\alpha_{1}$ and $\alpha_{2}$ ) with the measured fMRI BOLD activity. For lower $\beta_{1}$ and $\beta_{2}$, memory and planning capacity accordingly is more limited. We regressed the information values for different degrees of boundedness with the predicted information in the not-bounded case, where $\beta_{1}$ and $\beta_{2}$ were chosen high $\left(\beta_{1}=500\right.$, $\left.\beta_{2}=500\right)$. 
A

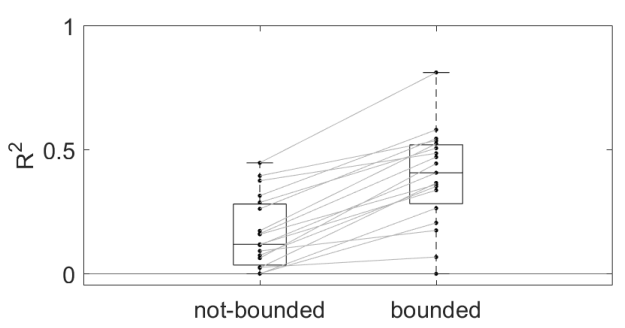

B

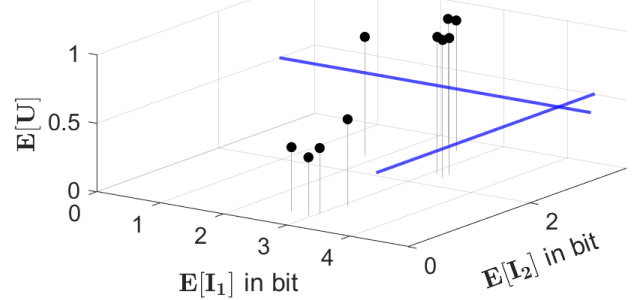

SPLI
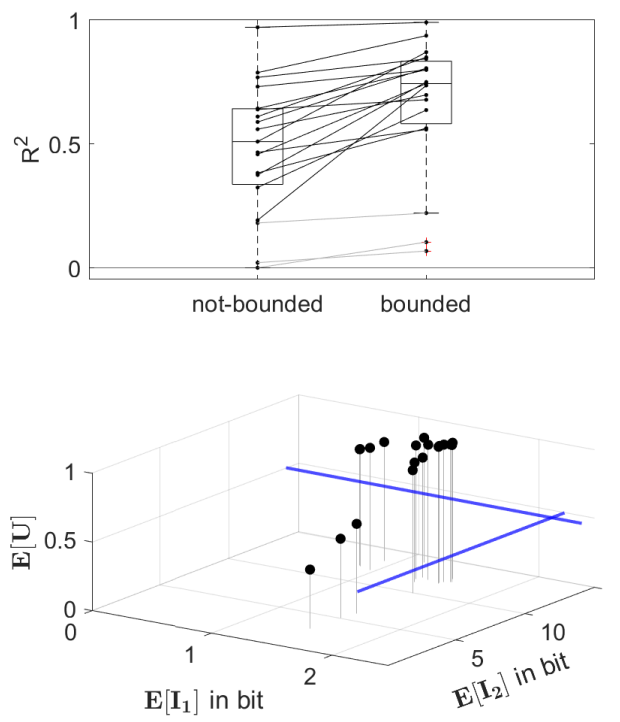

Figure 5: Model comparison. (A) $R^{2}$-values of the regressions for the best fitting information capacities for individual subjects for $\mathrm{H} 0$ and $\mathrm{H} 1$ are compared to not-bounded models. (B) Resulting theoretical quantitative predictions of planning effort for the different conditions of the experiment are plotted for individual subjects. Expected information $E\left[I_{1}\right]$ and $E\left[I_{2}\right]$ based on the fMRI correlates was lower than for not-bounded model (blue lines). This gives hint to prospective planning activity with subject-individual planning capacities.

$3 \mathrm{~d}$ graphs shown in Figure $5 \mathrm{~B}$. The expected information reductions over all conditions $E\left[I_{1}\right]$ and $E\left[I_{2}\right]$ of subject-individual best model fits were lower compared to the predicted information boundaries $E\left[I_{1}\right]$ and $E\left[I_{2}\right]$ when assuming no bounded information capacities for $I_{1}$ and $I_{2}$ (blue lines). Our comparison of the expected information reductions for the bounded and not-bounded model indicated, that for all subjects mainly information capacity for prospective planning $I_{2}$ was reduced whereas information for memory $I_{1}$ was reduced but still closer to the predictions of models with the not-bounded assumption. Expected information values for subject-specific model fits to activtiy in all other ROIs can be found in the Supplementary Material (Table 1 - Table 4). Assuming a simple $0 / 1$-utility function $U$ for our task (see Methods), the expected utility $E[U]$, shown on the z-axis, was individually different for the decision-making models and is higher for subjects with higher capacity limits for $I_{1}$ and $I_{2}$.

To finally investigate the impact of limited information processing for prospective planning in individual task conditions with different complexity (i.e. number of targets and number of movement steps), we illustrated frequencies of subjects' fitted information reduction in each condition in histogram representation in Figure 6. We contrasted the frequency distributions with the information predictions for a perfect decision-maker without information processing bounds (blue line). Deviations from the information with maximal capacities show evidence for bounded planning. It can be seen that subjects generally lie below the information bound of the perfect decision-maker. 
$\mathrm{H} 1$
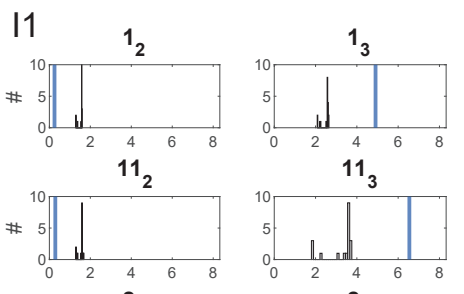

$11_{3}$
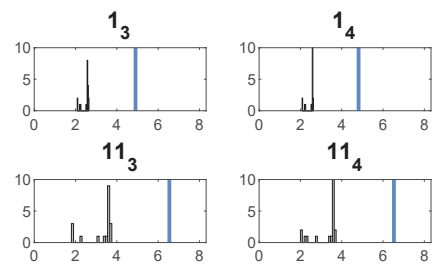

SPLI
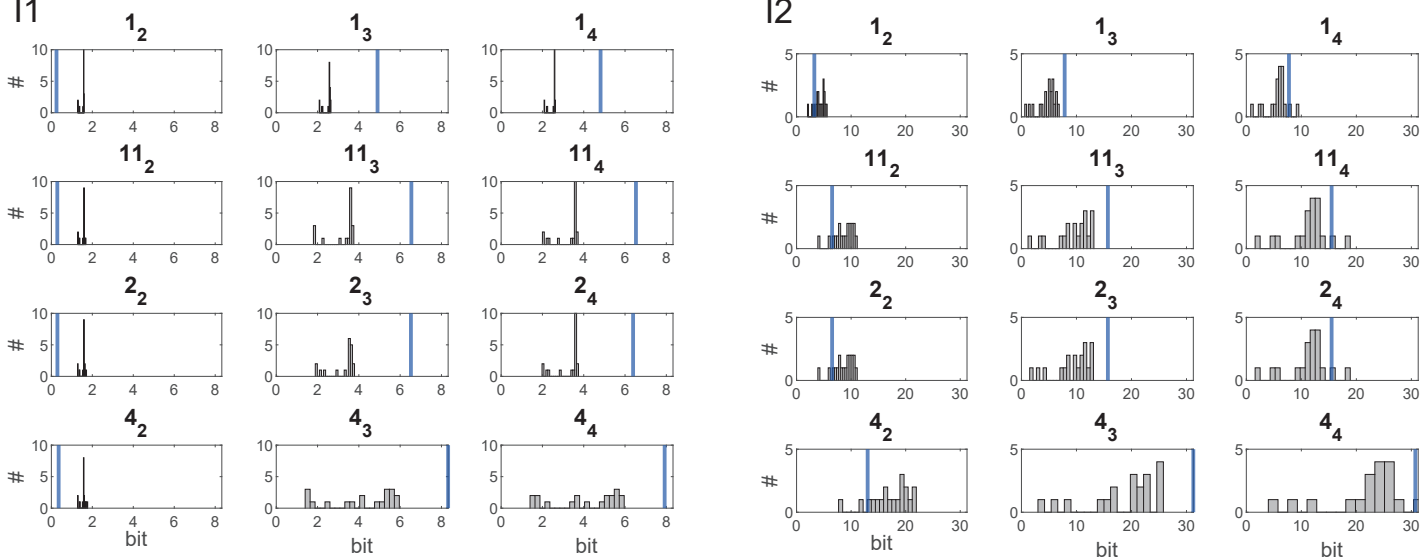

$4_{4}$
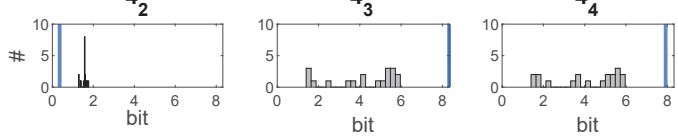

Figure 6: Theoretical expected information cost $I 1$ (left) and $I 2$ (right) of prospective planning for all individual subjects, in a histogram representing the frequency distribution. Condition dependent information varied between bounded model predictions regressed to subjects fMRI data compared to the model predictions in the not-bounded case (in blue). Note that generally subjects lie below the information bound of the not-bounded decision-maker and only for the most simple 2-step conditions subjects' information costs for memory (I1) deviate in the different direction from the predictions in the not-bounded case. This results from the specific task design and the fact, that bounds are provided on the total expected information. Decision-makers with not-bounded planning capacities can optimally assign resources to more difficult conditions and save memory costs for 2-step conditions, because no uncertainty about the target location will remain when $s_{2}$ is revealed given that all actions are planned prospectively under a high information resource for $I 2$. 


\section{Discussion}

Using fMRI and information-theoretic modeling, we have investigated neural correlates of information processing costs during concurrent motor planning in the human motor system, in particular in the planning-relevant brain areas including SPL, PMd, antIPS [15]. We manipulated informational complexity of movement planning by varying both the temporal extension of the movement sequences (the number of movement steps) as well as the degree of concurrency (the number of potential targets). We compared how much of the variance of the fMRI signal could be explained when assuming concurrent prospective planning for multiple potential targets (hypothesis $\mathrm{H} 1$ ) and when assuming no prospective planning, but only uncertainty reduction based on past sensory information (hypothesis $\mathrm{H} 0$ ). We found that the information cost of concurrent planning explained fMRI profiles across conditions significantly better than assuming a simple memory hypothesis that defers all anticipatory planning activity into the future when sensory stimuli resolve the remaining uncertainty. Our results therefore add to a growing body of evidence that suggests the use of concurrent prospective planning in the motor system when faced with multiple potential futures $[18,33,7,8,19,20,9,2,34,35,12,36,37,12,13,14]$.

While the parallel planning of multiple potential futures comes with a reaction time advantage whose behavioral consequences could be positively selected by evolution, it is also immediately clear that it cannot scale to scenarios with arbitrarily large amounts of uncertainty due to an excessive computational burden. Therefore, one would naturally expect a trade-off between the degree of concurrency in motor planning and the amount of information processing required. Evidence for such a trade-off has been previously reported in behavioral studies [38], where it has been shown that competition between movement plans increases motor variability, thus suggesting a shared resource for movement planning. In our experiment, this could be seen both in the behavior and the fMRI BOLD signals. Behaviorally, we noted an increased error rate for conditions with high degree of concurrency, suggesting a limitation in precision for concurrent planning. Neurally, we found that the best model correlates are characterized both by the assumptions of concurrent planning and limited information capacity. In particular, we found in our nested model analysis for the two information profiles regarding memory and planning that adding the planning information significantly improved the variance explained in the fMRI signal.

Serial vs. parallel processing The null hypothesis $\mathrm{H} 0$ has been aligned with more traditional theories of sensorimotor processing whose point of view is that perceptual, cognitive and motor processes take place successively and only a single motor program is prepared in the end $[5,6]$. For our experiment that means that the delay phase could only reduce uncertainty about the specification of that motor program to a certain extent based on the memory of the pre-cued information, so that the full specification of the motor program would have to be postponed until the end of the delay phase. Since the presence of more possibilities goes hand in hand with less information reduction, this hypothesis would predict less planning activity in multi-target conditions, whereas the concurrent planning hypothesis would predict increased activity in multi-target conditions. A direct comparison with fMRI activity is, however, confounded by persistent activity related to memory in both cases. Avoiding such a memory confound by showing the stimulus directly, would instead have produced a stimulus confound. Accordingly, we have conducted a bivariate regression of the fMRI signal for the expected amount of information processing for memory and planning. This regression analysis significantly 
favoured the concurrent planning hypothesis $\mathrm{H} 1$.

Previous neural studies that have favored the hypothesis of concurrent motor planning have been mostly conducted in monkeys. Cisek and colleagues [33] have found, for example, that PMd activity in a two-target condition reflects presence and relative location of both targets, and that once the right target is indicated by a nonspatial cue, the corresponding directional signal is increased, while the other one is suppressed, thus, suggesting two concurrent motor plans vying for execution. Similarly, Klaes and colleagues [9] have found that neurons in the frontoparietal reach areas of monkeys simultaneously represent two different movements associated with two different sensor-action rules, when it was made unclear to the monkey which of the rules to apply. In a similar vein, Cui and colleagues [2] have found in a nonspatial effector choice task (saccade vs reach) that the parietal reach region encodes both potential action plans, whereas the dorsal area $5 \mathrm{~d}$ only reflected the ultimately selected effector. A recent fMRI study [34] also reported supportive evidence for the affordance competition hypothesis in humans, demonstrating that task-relevant information biases activity of primary motor regions before movement onset and that coupling with fronto-parietal regions increased when the evidence for competing actions was similar. Other studies that have supported the affordance competition hypothesis in humans have mostly concentrated on behavioral features suggestive of concurrent planning $[35,12]$.

Other studies $[1,39,40]$ have argued that decisions between different options are made upstream in prefrontal brain areas and are independent of the particular sensorimotor contingency of the task, arguing against the notion of concurrent action planning. However, decisions in these tasks usually involve choices between goods (e.g. apple vs. banana), where the effector is not directly relevant for the choice, even though different action costs can be integrated within the valuation of different options [39]. This is in contrast to action-based decisions like in our study, where the choice is tightly linked with the effector through the spatial nature of the stimuli. Our study clearly falls in the latter category and fits with previous results on concurrent planning. In how far these results can be generalized to decisions between nonspatial stimuli with different values is an open question that might require the careful comparison of different stimulus and task designs.

Limitations in memory and planning In our study we have investigated limitations of concurrent planning in terms of the number and the sophistication of multiple plans. Limitations in parallel motor performance have been previously studied in dualtask designs. Considering the cognitive costs of dual task performance, serial processing frameworks $[36,37]$ were originally proposed that aim to explain delayed reaction times for secondary tasks [41]. Accordingly, the Response Selection Bottleneck (RSB) model [42] assumes that the crucial limitation (or bottleneck) in dual-task performance is located in the response selection stage. In contrast, capacity sharing models [43, 44] allow for sharing of cognitive resources in the central processing stages of two tasks. By manipulating task features like stimulus onset asynchrony and no-go trials, it has also been argued that subjects can somewhat switch between serial and parallel processing strategies $[45,46,47,48,49]$.

As demonstrated in the current study, our information-theoretic model can assume both serial and parallel information processing constraints and could be adapted to either scenario to allow for a quantitative analysis. Another important limitation is imperfect memory of the stimulus during action planning. The concept of working memory as a short-term active storage of information [50] relates to the idea of a limited capacity control process involved in keeping or discarding information [51]. Quantifying the limited 
information capacity of working memory has been addressed in numerous studies, for example on visual working memory $[21,23,24,25,52,26]$ and spatial working memory $[28,29,53,54]$ with growing evidence that they share a common resource limitation $[55$, 56]. In terms of neurophysiology, it has been suggested early on, that memory limitations can be considered as the result of the limited computational capacity of single neurons [57]. In our model, we have represented limitations of working memory more abstractly, by an internal variable that can only assume a finite amount of values and whose precision is curtailed by a corresponding information constraint. This abstraction is based on the idea that any information processing limitation can ultimately be thought of as a limitation in the amount of uncertainty that can be reduced [58].

Information theory, behavior and neural signals Our study belongs to a large family of investigations that have used information theory to quantitatively model information processing constraints in behavior and neural signals. In behavioral experiments information capacity limits have been established for working memory, attention, perceptual identification, decision-making, motor planning and execution [21, 59, 60, 30]. Informational complexity in behavioral tasks is often related to reaction or movement times and task accuracy. The most well-known examples of this are Hick's law that relates reaction time linearly to the entropy of a choice stimulus and Fitts' law that relates movement time to an index of difficulty measured in bits [61,62]. However, similar approaches have also been used to estimate the capacity for cognitive control depending on the entropy of the stimulus [63]. In our task, reaction times and error rates increased with higher task complexity, as expected from previous studies on motor sequence learning [3]. However, directly relating this change in reaction time to information complexity of our two hypotheses is confounded, because it is not clear whether the increase in reaction time during the response phase stems from a more complex decision between different readymade motor plans (H1) or whether the increase results from finishing a single motor plan based on the incomplete uncertainty reduction during the delay phase (H0).

Importantly then, by going beyond the behavioral analysis, we could use the different information profiles for concurrent and serial planning to regress the fMRI signal. However, simply applying information-theoretic concepts to brain signals without taking into account the behavioral task, would not have been enough to distinguish between the two hypotheses. Previous neurophysiological studies have, for example, used mutual information between stimuli/behavior and neural signals to establish a neural code either for encoding or decoding [64], quantified the richness of conscious states within a theory of integrated information [65], or related activity in distinct regions of the prefrontal cortex to sensorimotor and cognitive control [66, 67]. A considerable number of imaging studies have also found neural correlates of informational surprise in sensory areas of the brain $[68,69,70]$ related to prediction error in the context of predictive coding theory, with a wide range of applications ranging from action understanding in the mirror neuron system [71, 72, 73] and value-based decision-making [74] to hierarchical motor control [75]. However, in order to go beyond information-theoretic quantification of neural signals we need to consider both information constraints and task-related constraints [76].

Bounded rationality In contrast to many previous studies that have relied on information theory to estimate neural processing capacity, we have employed a class of bounded rationality models that trade off both utility and information processing costs $[77,78,79,80,81]$, similar to the rate distortion trade-off in information theory [82] that defines relevant information when bandwidth is limited. Such a generalized trade-off 
makes this class of models applicable to tasks with arbitrary utility function. Moreover, using information constraints on multiple variables, we can design optimality models that simultaneously explain behavior and optimal information flow between internal variables that can be correlated with fMRI BOLD signals. In previous behavioral studies, the trade-off between utility and information has been used in the context of rational inattention and quantal response equilibria in economic decision-making [83, 84], perceptual representation in identification and memory tasks $[60,85]$, reaction time and endpoint variability for motor planning under time constraints [30], abstraction in sensorimotor processing [31], decision-making by sampling [86] and planning in a Markov environment $[87,88]$.

Similar to these previous studies where behavioral task performance and information processing capacities were measured, we here use a normative probabilistic optimality model for uncertainty reduction and parallel processing. In this study, however, we go a step further and relate the predicted information flow with fMRI activity during motor planning. In particular, measuring the activity of premotor and parietal planning areas during the delayed response tasks allowed us to measure planning capacity for movement preparation. As the bounded rationality framework allows for multiple information constraints including internal variables for memory and action planning, we could regress fMRI activity with respect to these different information quantities and we could test the hypothesis of concurrent prospective planning (H1) against the null hypothesis of mere uncertainty reduction (H0). This approach rests on the assumption that brain signals reflect at least approximately an optimal information flow - an assumption that has been applied very successfully in the past, for example in the context of sparse coding [89, 90]. Ultimately, it is an empirical question for future studies, how far this class of models can be developed in their explanatory power, but they open a new exciting avenue that allows relating both behavior and neural representations to optimal information processing assumptions.

\section{Acknowledgments}

This study was funded by European Research Council (BRISC: Bounded Rationality in Sensorimotor Coordination).

\section{Author contributions}

S.S., A.L. and D.A.B. designed the experiment; S.S. and A.L. developed the experimental setup and acquired data; S.S., D.A.B. generated theoretical predictions from computer simulations; A.L., D.A.B supervised the project; S.S., A.L. and D.A.B. wrote, discussed and edited the manuscript.

\section{Declaration of interests}

The authors declare no competing interests. 


\section{Methods}

\section{Experimental Methods}

Subjects Nineteen right-handed healthy subjects (11 females, 8 males) in the age range of 22 and 41 years (mean $=27.5, \mathrm{SD}=4.5$ ) participated in the experiment and were included in further data analysis. Initially, we recruited 22 subjects, but data of three subjects was excluded because of strong movement artifacts. Participants provided written informed consent in accordance with the declaration of Helsinki and the study was approved by the ethics committee of the University Hospital and the Faculty of Medicine at the University of Tübingen.

Experimental Paradigm. We employed a delayed response task (DRT), in which subjects were instructed to generate sequences of key presses with four possible keys to control right, left, up, and down of a cursor movement. Each trial started with a random fixation phase of $13.5-16$ sec (FIX), where a fixation cross was presented in the middle of the four fields and should be fixated with the eyes. This fixation period was followed by three principle task task phases: cue presentation, delay/planning and response. In the cue presentation phase, subjects were shown one or multiple potential targets (four different target planning conditions $c_{T} \in\left\{{ }^{\prime} 1\right.$, ' '11', '2', '4' $\}$ as detailed below) with one of three possible target distances (three different step conditions $c_{S} \in\{2,3,4\}$ ). The timeline of a DRT is illustrated in Figure 1.

Cue presentation phase. The display area is divided into four field panels each made up of $3 \times 5$ fields as shown in Figure 1 A). Each field represents a target position that can be reached with a cursor. Panels could be surrounded by a frame, which indicated that the highlighted target position within the frame should be taken into consideration as a potential target for motor planning. The potential target positions were always shown for 3 sec (CUE). Depending on the target planning condition, there were four different kinds of cues:

- '1'-Target Planning Condition: In this one-fold planning condition only one panel was framed containing a single highlighted potential target location that always corresponded to the actual target location in the response phase.

- '11'-Target Planning Condition: In this two-fold planning condition there was also a single framed panel that contained two potential targets highlighted, only one of which was selected as the actual target during response phase.

- '2'-Target Planning Condition: In this two-fold planning condition, two panels were framed, each containing a single highlighted potential target from which one was selected for execution.

- '4'-Target Planning Condition: In this four-fold planning condition all four panels were framed, each containing a single potential target highlighted for planning, from which one was selected in the response phase.

Cue presentation was followed by a mask of 1 sec (MASK), which was intended to "overwrite" any after-images of the cue.

Planning phase. The planning phase was a delay phase of $14-16.5$ sec (DEL) following the mask to allow subjects to plan one or multiple movement paths based on the information they obtained from the visual target cues. During this time, only a fixation 
cross was displayed in the center of the screen. From trial start to the completion of the planning phase, subjects had to keep their finger in rest position and where therefore asked to press the central button of the input device.

Response phase. In the response phase (RES), the actual target was determined from the set of potential targets. A half-size-frame encompassing six fields (including the actual target) was displayed around one half of one of the four field panels, however, the correct target itself was not highlighted in any way. Accordingly, the correct target field could only be uniquely identified if subjects remembered the appropriate information from the previously displayed target cues. Subjects were instructed to perform the movement sequence towards the inferred target field as fast and directly as possible within the allowed time window of $3 \mathrm{sec}$ (otherwise the trial was terminated with an incomplete response). Depending on the distance of the target (step condition), only the first 2, 3 or 4 key presses were considered before the trial was terminated. Subjects could observe the corresponding cursor movement and got immediate visual feedback of their performance at the end of the trial, by highlighting the cursor position in green (red) when the reached position was correct (incorrect).

Besides DRT trials, we also included control trials in which no planning was required/ possible during the delay phase. Like the other DRT trials, control trials were comprised an initial fixation phase and the three principle task phases - cue presentation, planning and response. Different to the DRT, however, in the cue presentation phase, no targets were highlighted. Hence, subjects were not informed about any potential target location and therefore could not plan specific movements during the delay planning phase. In the response phase, the actual target was indicated directly by highlighting the corresponding target field, so that the movement sequence to reach the target could only be planned once the actual target location was revealed.

Our experiment usually consisted of four blocks of 30 trials each (one subject had five blocks, two subjects only three blocks) resulting in a total of 120 trials and lasting $\sim 80$ min per subject. Each block consisted of 6 control trials and 6 trials for each target planning condition. Moreover, the 6 trials had an equal share of step conditions, i.e. 2 trials with target distance 2,3 , and 4 steps, respectively. The order of the trials in any given block of 30 was permuted randomly.

In our experimental setup we used Windows based computers with the softwares Matlab R2007b (The MathWorks, Inc.) and Cogent Graphics (developed by John Romaya at the LON at the Wellcome Department of Imaging Neuroscience) to generate the visual stimuli for the experimental trial sequences and to record and store subjects' behavioral responses and all relevant information associated with the visual stimuli sequence. The visual stimuli were projected to a screen, using an LCD projector (1024x768 pixels; $60 \mathrm{~Hz}$ refresh rate), and presented to the subjects from the display behind the head coil of the scanner with a mirror with a size $28 \mathrm{deg} \times 37 \mathrm{deg}$ visual angle of the projected image.

Behavioral performance monitoring The instructed finger movements were monitored using the 5 Button Diamond Fibre Optic response device (Current Design, Philadelphia, US) operated by repetitive thumb flexions. From this we could extract reaction and movement times as well as error rates defined by the frequency of target misses. For behavioral analysis, we excluded trials with undefined reaction and movement times, when movements were not initiated or completed. Additionally, eye movements were monitored at $50 \mathrm{~Hz}$ sampling rate throughout the scanning sessions using an MRI-compatible eye camara and infra-red illumination system (SMI SensoMotoric Instruments) and Viewpoint Eye Tracker software (Arrington Research, Scottsdale, US). Eye position recordings were 
filtered using a $10 \mathrm{~Hz}$ Chebyshev Type II low-pass filter. Saccades were detected using an absolute velocity threshold of 30 degree per second (for a single recording of one subject 40 degree per second) and saccade frequency per second reported on average over subjects during delay. Eye measures from 2 subjects were excluded from the analysis because of incompleteness.

We performed a 2-way repeated measures ANOVA (rmANOVA) with the factor target condition ('1', '11', '2', '4' and CT) and factor steps (2, 3, or 4 steps) and applied post-hoc pairwise rmANOVAs for the behavioral analysis of reaction times, movement times, error rates and saccades. For this and all other rmANOVAs we tested for sphericity (Mauchly's test) and applied the Greenhouse-Geisser correction whenever the assumption of sphericity was violated.

MRI image acquisition and analysis MRI images were aquired on a $3 \mathrm{~T}$ Siemens PRISMA scanner (Siemens, Ellwangen, Germany). A T1-weighted magnetization prepared rapid-acquisition gradient echo (MP-RAGE) structural scan (176 slices, 256x256 voxel resolution, $1 \mathrm{x} 1 \mathrm{~mm}$ inplane voxel size, slice thickness $=1 \mathrm{~mm}$, gap $=0 \mathrm{~mm}$, repetition time $=2300 \mathrm{~ms}$, echo time $=2.96 \mathrm{~ms}$, field of view $=256 \mathrm{~mm}$ ) was obtained in parallel to the task training. Functional T2*-weighted gradient-echo planar imaging (EPI) volumes (48 sclices, 64 voxel resolution, $3 \times 3 \mathrm{~mm}$ inplane voxel size, slice thickness $=3 \mathrm{~mm}$ ,gap $=0 \mathrm{~mm}$, repetition time $=2000 \mathrm{~ms}$, echo time $=35 \mathrm{~ms}$, field of view $=1344 \mathrm{~mm}$, flip angle $=75 \mathrm{deg}$ ) completely covered the the cerebral cortex as well as most subcortical structures. Per subject we obtained 600 EPIs during a single run of 19.75 min length, resulting overall in 2400 EPIs for 18 of the subjects (1800 and 2177 for prematurely terminated recordings and 3000 for a subject with an extra recorded run). Functional image processing was conducted with the SPM12 software package (Wellcome Centre for Human Neuroimaging, London, UK, Matlab 2016a Release) and included spatial realignment of all functional images to the first EPI image as a reference, co-registration of the anatomical T1 image and the mean functional EPI image, spatial normalization to the Montreal Neurological Institute space (MNI-template) with voxel size of $1 \times 1 \times 1 \mathrm{~mm}^{3}$ for $\mathrm{T} 1$ and $3 \times 3 \times 3 \mathrm{~mm}^{3}$ for EPI images and spatial smoothing of the normalized EPIs with a Gaussian kernel ( $7 \mathrm{~mm}$ full-width at half maximum (FWHM) Gaussian filter.

Functional fMRI analysis was first performed on an individual level (first-level analysis) and then on the group level (second-level analysis). The subject-specific analysis of fMRI BOLD activity is based on a general linear model (GLM), where we specified model regressors for the cue phase (CUE) for each target planning conditions and regressors for the delay and response phase (DEL, RES) for each of the 4 target planning conditions $\left(c_{T}\right)$ and the control condition combined with the three step conditions $\left(c_{S}\right)$. In addition the 6 motion parameters from the realignment procedure were additionally included as regressors of no interest. The fixation phase was not explicitly modeled and served as an implicit baseline. Each experimental session therefore was modelled separately with 41 regressors for each subject. Finally, all regressors were convolved with the canonical hemodynamic response function.

Regions of interest (ROIs) were selected as brain areas that exhibited significant planning-related activity in the DRT conditions compared to the control-task across subjects. Given the estimated regressor parameters of the GLM, in the 2nd-level analysis, we first determined significant voxels whose t-values for the contrast of interest $\left(\mathrm{DRT}_{\text {Delay }}\right.$ $>\mathrm{CT}_{\text {Delay }}$ ) were below a threshold of $p<0.05$ (t-test across subjects' first level contrast images for $\mathrm{DRT}_{\text {Delay }}>\mathrm{CT}_{\text {Delay }}$ family-wise error FWE-corrected for multiple comparisons). We found significant bilateral activities in brain areas typically involved in motor 
Bounded Rationality Model We applied the information-theoretic bounded rationality framework [91, 92] to our experimental task, where information about the hidden world state $w \in \mathcal{W}$ (the exact target location) is revealed in two steps, by first revealing the combined cue stimulus $s_{1} \in \mathcal{S}_{1}$ indicating all possible target positions, and then the second stimulus $s_{2} \in \mathcal{S}_{2}$ that resolves the remaining uncertainty about the actual target $w$. After the combined cue stimulus, the agent can form a memory $m \in \mathcal{M}$ before perceiving the second stimulus $s_{2}$ and selecting an action $a \in \mathcal{A}$ that corresponds to a movement path. The sets $\mathcal{W}, \mathcal{S}_{1}, \mathcal{S}_{2}, \mathcal{M}$ and $\mathcal{A}$ are finite and discrete, with $|\mathcal{W}|=40$ possible target locations, $|\mathcal{A}|=80$ possible movement paths, $\left|\mathcal{S}_{2}\right|=8$ possible half-sized frames for each panel and $\left|\mathcal{S}_{1}\right|=|\mathcal{M}|=820$ possible combinations of potential targets over all conditions. For any particular target condition $c_{T}$ and step condition $c_{S}$, we then get a uniform distribution over stimuli

$$
p\left(s_{1} \mid c_{T}, c_{S}\right)= \begin{cases}\frac{1}{N_{c_{T}, c_{S}}} & \text { if } s_{1} \in \mathcal{S}_{c_{T}, c_{S}} \subset \mathcal{S}_{1} \\ 0 & \text { otherwise }\end{cases}
$$


where $\mathcal{S}_{c_{T}, c_{S}}$ is the subset of stimuli that belong to the condition $\left(c_{T}, s_{T}\right)$ and $N_{c_{T}, c_{S}}=$ $\left|\mathcal{S}_{c_{T}, c_{S}}\right|$ is the number of stimuli in that condition, where $N_{1,2}=8, N_{1,3}=16, N_{1,4}=16$, $N_{11,2}=4, N_{11,3}=16, N_{11,4}=16, N_{2,2}=24, N_{2,3}=96, N_{2,4}=96, N_{4,2}=16$, $N_{4}, 3=256$, and $N_{4,4}=256$. The marginal distribution over stimuli is given by $p\left(s_{1}\right)=$ $\sum_{c_{T}, c_{S}} p\left(c_{t}, c_{S}\right) p\left(s_{1} \mid c_{T}, c_{S}\right)$ where all conditions are equally likely with $p\left(c_{T}, c_{S}\right)=\frac{1}{12}$. As the second stimulus $s_{2}$ surrounds any potential target from $s_{1}$ with a rectangular halfframe, the distribution $p\left(s_{2} \mid s_{1}\right)$ is a uniform with non-zero probability over a single value of $s_{2}$ when $c_{T}=$ '1', two possible values of $s_{2}$ when $c_{T}=$ '11' or $c_{T}=' 2$ ', or four possible values of $s_{2}$ when $c_{T}=' 4$ '.

In the model, the agent chooses the action $a$ to maximize the task utility $U(w, a)$ under the constraint that only a certain amount of information-processing can be achieved when forming the memory $m$ and selecting the action $a$. For our task the utility function $U$ is a simple 0/1-utility: it is 1 whenever the action $a$ is compatible with target $w$ and 0 otherwise. Since information-processing can be unreliable, the agent's memory and decision are formalized by probability distributions $p\left(m \mid s_{1}\right)$ and $p\left(a \mid m, s_{2}\right)$, such that the amount of information processing can be captured by the Kullback-Leibler divergence between the prior distributions $p(m)$ and $p(a)$ and the posterior distributions $p\left(m \mid s_{1}\right)$ and $p\left(a \mid m, s_{2}\right)$, respectively. The bounded rational decision-making problem can then be written as a constrained optimization problem

$$
\max \left\{p\left(a \mid m, s_{2}\right), p\left(m \mid s_{1}\right) E[U]-\frac{1}{\beta_{1}} I\left(M, S_{1}\right)-\frac{1}{\beta_{2}}\left(I(A ; M)+I\left(A ; S_{2} \mid M\right)\right)\right\} .
$$

where the expectation is taken with respect to the distribution

$$
p\left(w, s_{1}, s_{2}, m, a\right)=p\left(w \mid s_{1}, s_{2}\right) p\left(s_{1}\right) p\left(s_{2} \mid s_{1}\right) p\left(m \mid s_{1}\right) p\left(a \mid m, s_{2}\right),
$$

with $p\left(s_{1}\right), p\left(s_{2} \mid s_{1}\right)$ and $p\left(w \mid s_{1}, s_{2}\right)$ defined by the task, and $p\left(m \mid s_{1}\right)$ and $p\left(a \mid m, s_{2}\right)$ left for optimization. The information quantities $I\left(M, S_{1}\right)$ and $I(A ; M)+I\left(A ; S_{2} \mid M\right)$ respectively measure the average Kullback-Leibler divergence between the distributions $p(m)$ and $p(m \mid s 1)$ for memory formation and the average Kullback-Leibler divergence between the distributions $p(a)$ and $p\left(a \mid m, s_{2}\right)$ for generating a specific action given memory $m$ and stimulus $s_{2}$. The parameters $\beta_{1}$ and $\beta_{2}$ reflect the degree of boundedness, where $\beta_{1,2} \rightarrow \infty$ reproduces a Bayes-optimal maximum expected utility decision-maker. The bounded optimal solution for equation 2 is given by

$$
\left\{\begin{array}{l}
p\left(m \mid s_{1}\right) \propto p(m) e^{\beta_{1} \Delta F\left(m, s_{1}\right)} \\
p(m)=\sum_{s_{1}} p\left(s_{1}\right) p\left(m \mid s_{1}\right) \\
p\left(a \mid m, s_{2}\right) \propto p(a) e^{\beta_{2} \sum_{w} p\left(w \mid m, s_{2}\right) U(w, a)} \\
p(a)=\sum_{s_{1}, s_{2}, m} p\left(a \mid m, s_{2}\right) p\left(m \mid s_{1}\right) p\left(s_{2} \mid s_{1}\right) p\left(s_{1}\right)
\end{array}\right.
$$

with

$$
\Delta F\left(m, s_{1}\right)=\sum_{a, s_{2}, w} p\left(a \mid m, s_{2}\right) p\left(s_{2} \mid s_{1}\right) p\left(w \mid s_{1}, s_{2}\right) U(w, a)-\frac{1}{\beta_{2}} \sum_{a, s_{2}} p\left(a \mid m, s_{2}\right) p\left(s_{2} \mid s_{1}\right) \log \left(\frac{p\left(a \mid m, s_{2}\right)}{p(a)}\right)
$$

and with $p\left(w \mid m, s_{2}\right)$ determined from equation 3 .

Modelling information-processing during Delay Phase. If we want to apply equation (4) to model planning during the delay phase, we have to be aware that the decision rule $p\left(a \mid m, s_{2}\right)$ requires the agent to know the second stimulus $s_{2}$ when deciding 
about the action $a$, an information that is not available during the delay period. We consider two hypotheses.

- Hypothesis 0: Planning of the action $a$ according to $p\left(a \mid m, s_{2}\right)$ is delayed until the execution phase, once $s_{2}$ is known. The delay phase is only used for uncertainty reduction, modeled by the mathematical transition from the prior $p(m)$ to the posterior $p\left(m \mid s_{1}\right)$ and in the space of actions from $p(a)$ to $p(a \mid m)$. In any particular condition $\left(c_{T}, c_{S}\right)$, we would then expect the information costs

$$
\begin{aligned}
& I_{1}\left(c_{T}, c_{S}\right)=\sum_{s_{1}} p\left(s_{1} \mid c_{T}, c_{S}\right) \sum_{m} p\left(m \mid s_{1}\right) \log \frac{p\left(m \mid s_{1}\right)}{p(m)} \\
& I_{2}\left(c_{T}, c_{S}\right)=\sum_{s_{1}} p\left(s_{1} \mid c_{T}, c_{S}\right) \sum_{m} p\left(m \mid s_{1}\right) \sum_{a} p(a \mid m) \log \frac{p(a \mid m)}{p(a)}
\end{aligned}
$$

for memory formation and action processing respectively, and the average over all conditions given by $\sum_{c_{T}, c_{S}} p\left(c_{T}, c_{S}\right) I_{1}\left(c_{T}, c_{S}\right)=I\left(M ; S_{1}\right)$ and
$\sum_{c_{T}, c_{S}} p\left(c_{T}, c_{S}\right) I_{2}\left(c_{T}, c_{S}\right)=I(A ; M)$

- Hypothesis 1: Prospective Planning. Once the uncertainty over actions is reduced to $p(a \mid m)$ by observing $s_{1}$, all possible $s_{2}$ are anticipated in the delay phase, and for each $s_{2}$ an action is planned according to $p\left(a \mid m, s_{2}\right)$. Depending on available information resources, the plans can be more or less precise. Once the actual stimulus $s_{2}$ is revealed during the response phase, one of the planned actions can be immediately carried out. During the delay phase in any particular condition $\left(c_{T}, c_{S}\right)$, we would then expect the information costs

$$
\begin{aligned}
& I_{1}\left(c_{T}, c_{S}\right)=\sum_{s_{1}} p\left(s_{1} \mid c_{T}, c_{S}\right) \sum_{m} p\left(m \mid s_{1}\right) \log \frac{p\left(m \mid s_{1}\right)}{p(m)} \\
& I_{2}\left(c_{T}, c_{S}\right)=N_{T, S} \sum_{s_{1}} p\left(s_{1} \mid c_{T}, c_{S}\right) \sum_{m} p\left(m \mid s_{1}\right) \sum_{s_{2}} p\left(s_{2} \mid s_{1}\right) \sum_{a} p\left(a \mid m, s_{2}\right) \log \frac{p\left(a \mid m, s_{2}\right)}{p(a)}
\end{aligned}
$$

for memory formation and action processing respectively, with $N_{1, S}=1^{2}, N_{11, S}=2^{2}$, $N_{2, S}=2^{2}$ and $N_{4, S}=4^{2}$, and the average over all conditions given by $\sum_{c_{T}, c_{S}} p\left(c_{T}, c_{S}\right) I_{1}\left(c_{T}, c_{S}\right)=I\left(M ; S_{1}\right)$ and $\sum_{c_{T}, c_{S}} \frac{1}{N_{T, S}} p\left(c_{T}, c_{S}\right) I_{2}\left(c_{T}, c_{S}\right)=I(A ; M)+I\left(A ; S_{2} \mid M\right)$.

Assuming a linear relationship between informational surprise and brain signal $\operatorname{fMRI}\left(c_{T}, c_{S}\right)$ for each condition $\left(c_{T}, c_{S}\right)$, we get a linear regression model

$$
\mathrm{fMRI}\left(\mathrm{c}_{\mathrm{T}}, \mathrm{c}_{\mathrm{S}}\right)=\alpha_{1} I_{1}\left(c_{T}, c_{S}\right)+\alpha_{2} I_{2}\left(c_{T}, c_{S}\right)+\alpha_{0},
$$

with model parameters $\alpha_{i}, i=0,1,2$.

For each of the two hypothesis $\mathrm{H} 0$ and $\mathrm{H} 1$, we tested the multilinear regression between the fMRI activity modulation and the two information modulations predicted by the models with a nested model F-statistic $(\alpha=5 \%)$, to find if $I_{2}$ significantly improves the regression. Therefore, we applied the statistical test to the models with subject's individual best fitting capacities and for individual fMRI activities in all relevant ROIs. 


\section{References}

[1] Padoa-Schioppa C. Neurobiology of economic choice: a good-based model. Annual review of neuroscience. 2011;34:333-59.

[2] Cui H, Andersen RA. Different Representations of Potential and Selected Motor Plans by Distinct Parietal Areas. Journal of Neuroscience. 2011;31(49):18130-18136.

[3] Schmidt RA, Lee TD. Motor control and learning: A behavioral emphasis, 3rd ed. Champaign, IL, US: Human Kinetics; 1999.

[4] Mcclelland J. On the time relations of mental processes: An examination of systems of processes in cascade. Psychological Review. 1979;86:287-330.

[5] Miller GA, Galanter E, Pribram KH. Plans and the structure of behavior. New York, NY, US: Henry Holt and Co; 1960.

[6] Keele SW. Movement control in skilled motor performance. Psychological Bulletin. 1968;70(6, Pt.1):387-403.

[7] Cisek P. Cortical mechanisms of action selection: the affordance competition hypothesis. Philosophical transactions of the Royal Society of London Series B, Biological sciences. 2007;362:1585-99.

[8] Cisek P, Kalaska JF. Neural Mechanisms for Interacting with a World Full of Action Choices. Annual Review of Neuroscience. 2010;33(1):269-298.

[9] Klaes C, Westendorff S, Chakrabarti S, Gail A. Choosing goals, not rules: deciding among rule-based action plans. Neuron. 2011;70:536-48.

[10] Cisek P. Neural representations of motor plans, desired trajectories, and controlled objects. Cognitive Processing. 2005;6:15-24.

[11] Shadlen MN, Newsome WT. Neural basis of a perceptual decision in the parietal cortex (area LIP) of the rhesus monkey. Journal of neurophysiology. 2001;86:1916-36.

[12] Gallivan JP, Barton KS, Chapman CS, Wolpert DM, Randall Flanagan J. Action plan co-optimization reveals the parallel encoding of competing reach movements. Nature Communications. 2015;6(1):7428.

[13] Gallivan JP, Logan L, Wolpert DM, Flanagan JR. Parallel specification of competing sensorimotor control policies for alternative action options. Nature Neuroscience. 2016;19(2):320-326.

[14] Nashed JY, Crevecoeur F, Scott SH. Rapid Online Selection between Multiple Motor Plans. Journal of Neuroscience. 2014;34(5):1769-1780.

[15] Lindner A, Iyer A, Kagan I, Andersen RA. Human Posterior Parietal Cortex Plans Where to Reach and What to Avoid. Journal of Neuroscience. 2010;30(35):1171511725 .

[16] Wolpert DM, Landy MS. Motor control is decision-making. Current opinion in neurobiology. 2012;22:996-1003.

[17] Wu SW, Delgado MR, Maloney L. Motor Decision-Making. Brain Mapping: An Encyclopedic Reference. 2015;3:417-427. 
[18] Kim JN, Shadlen MN. Neural correlates of a decision in the dorsolateral prefrontal cortex of the macaque. Nature neuroscience. 1999;2:176-85.

[19] Beck JM, Ma WJ, Kiani R, Hanks T, Churchland AK, Roitman J, et al. Probabilistic Population Codes for Bayesian Decision Making. Neuron. 2008;60(6):1142-1152.

[20] Wang XJ. Decision Making in Recurrent Neuronal Circuits. Neuron. 2008;60(2):215234.

[21] Daneman M, Carpenter PA. Individual differences in working memory and reading. Journal of Verbal Learning and Verbal Behavior. 1980;19(4):450-466.

[22] Conway A, Kane M, Bunting M, Hambrick Z, Wilhelm O, Engle R. Working memory span task: A methodological review and user's guide. Psychonomic bulletin \& review. $2005 ; 12: 769-86$.

[23] Luck SJ, Vogel EK. The capacity of visual working memory for features and conjunctions. Nature. 1997;390(6657):279-281.

[24] Luck SJ, Vogel EK. Visual working memory capacity: from psychophysics and neurobiology to individual differences. Trends in cognitive sciences. 2013;17:391-400.

[25] Vogel EK, Awh E. How to Exploit Diversity for Scientific Gain: Using Individual Differences to Constrain Cognitive Theory. Curr Dir Psychol Sci. 2008;17(2):171-176.

[26] Wilhelm O, Hildebrandt A, Oberauer K. What is working memory capacity, and how can we measure it? Frontiers in psychology. 2013;4:433-433.

[27] Oberauer K. Handbook of Understanding and Measuring Intelligence. Thousand Oaks, California: SAGE Publications, Inc.; 2005.

[28] Crawford LE, Landy D, Salthouse TA. Spatial working memory capacity predicts bias in estimates of location. Journal of experimental psychology Learning, memory, and cognition. 2016;42(9):1434-1447.

[29] Zhang W, Luck SJ. The Number and Quality of Representations in Working Memory. Psychol Sci. 2011;22(11):1434-1441.

[30] Schach S, Gottwald S, Braun DA. Quantifying Motor Task Performance by Bounded Rational Decision Theory. Frontiers in Neuroscience. 2018;12:932.

[31] Lindig-León C, Gottwald S, Braun DA. Analyzing Abstraction and Hierarchical Decision-Making in Absolute Identification by Information-Theoretic Bounded Rationality. Frontiers in neuroscience. 2019;13:1230.

[32] Masson MEJ. "Using confidence intervals for graphically based data interpretation": Correction to Masson and Loftus (2003). Canadian Journal of Experimental Psychology/Revue canadienne de psychologie expérimentale. 2004;58(4):289-289.

[33] Cisek P, Kalaska JF. Neural correlates of reaching decisions in dorsal premotor cortex: specification of multiple direction choices and final selection of action. Neuron. 2005;45:801-14.

[34] Calderon CB, Van Opstal F, Peigneux P, Verguts T, Gevers W. Task-Relevant Information Modulates Primary Motor Cortex Activity Before Movement Onset. Frontiers in Human Neuroscience. 2018;12:93. 
[35] Chapman CS, Gallivan JP, Wood DK, Milne JL, Culham JC, Goodale MA. Reaching for the unknown: Multiple target encoding and real-time decision-making in a rapid reach task. Cognition. 2010;116(2):168-176.

[36] Schweickert R. A critical path generalization of the additive factor method: Analysis of a Stroop task. Journal of Mathematical Psychology. 1978;18(2):105-139.

[37] Pashler H. Graded capacity-sharing in dual-task interference? Journal of experimental psychology Human perception and performance. 1994;20:330-42.

[38] Oostwoud Wijdenes L, Ivry RB, Bays PM. Competition between movement plans increases motor variability: evidence of a shared resource for movement planning. Journal of Neurophysiology. 2016;116(3):1295-1303.

[39] Cai X, Padoa-Schioppa C. Neuronal evidence for good-based economic decisions under variable action costs. Nature Communications. 2019;10(1):393.

[40] Rushworth MFS, Kolling N, Sallet J, Mars RB. Valuation and decision-making in frontal cortex: one or many serial or parallel systems? Current opinion in neurobiology. 2012;22:946-55.

[41] Pashler H. The Psychology of attention. Cambridge, MA: The MIT Press; 1998.

[42] Pashler H. Dual-task interference in simple tasks: data and theory. Psychological bulletin. 1994;116:220-44.

[43] Navon D, Miller J. Queuing or sharing? A critical evaluation of the single-bottleneck notion. Cognitive psychology. 2002;44:193-251.

[44] Tombu M, Jolicoeur P. A Central Capacity Sharing Model of Dual-Task Performance. Journal of experimental psychology Human perception and performance. 2003;29:318.

[45] Fischer R, Gottschalk C, Dreisbach G. Context-sensitive adjustment of cognitive control in dual-task performance. Journal of experimental psychology Learning, memory, and cognition. 2014;40:399-416.

[46] Fischer R, Plessow F. Efficient multitasking: parallel versus serial processing of multiple tasks. Frontiers in Psychology. 2015;6:1366.

[47] Fischer R, Dreisbach G. Predicting high levels of multitasking reduces betweentasks interactions. Journal of experimental psychology Human perception and performance. 2015;41:1482-7.

[48] Lehle C, Steinhauser M, Hübner R. Serial or parallel processing in dual tasks: What is more effortful? Psychophysiology. 2009;46(3):502-509.

[49] Miller J, Ulrich R, Rolke B. On the optimality of serial and parallel processing in the psychological refractory period paradigm: effects of the distribution of stimulus onset asynchronies. Cognitive psychology. 2009;58:273-310.

[50] Atkinson RC, Shiffrin RM. Human Memory: A Proposed System and its Control Processes. In: Spence KW, Spence JT, editors. The psychology of learning and motivation: Advances in research and theory. vol. 2 of Psychology of Learning and Motivation. Academic Press; 1968. p. 89-195. 
[51] Baddeley AD, Hitch G. Working Memory. In: Bower GH, editor. Recent Advances in Learning and Motivation. vol. 8 of Psychology of Learning and Motivation. Academic Press; 1974. p. 47-89.

[52] Oberauer K, Farrell S, Jarrold C, Lewandowsky S. What limits working memory capacity? Psychological bulletin. 2016;142:758-99.

[53] Wood J. "A core knowledge architecture of visual working memory": Correction to Wood (2011). Journal of experimental psychology Human perception and performance. 2011;37:633.

[54] Woodman G, Luck S. Visual search is slowed when visuospatial working memory is occupied. Psychonomic bulletin \& review. 2004;11:269-74.

[55] D'Esposito M, Postle BR. The Cognitive Neuroscience of Working Memory. Annual Review of Psychology. 2015;66(1):115-142.

[56] Cowan N. The magical number 4 in short-term memory: A reconsideration of mental storage capacity. Behavioral and Brain Sciences. 2001;24(1):87-114.

[57] Fuster JM, Alexander GE. Neuron activity related to short-term memory. Science (New York, NY). 1971;173:652-4.

[58] Gottwald S, Braun DA. Bounded Rational Decision-Making from Elementary Computations That Reduce Uncertainty. Entropy. 2019;21(4).

[59] Verghese P, Pelli DG. The information capacity of visual attention. Vision Research. 1992;32(5):983-995.

[60] Sims CR. Rate-distortion theory and human perception. Cognition. 2016;152:181198.

[61] Hick WE. On the rate of gain of information. The Quarterly Journal of Experimental Psychology. 1952;4:11-26.

[62] Fitts PM, Peterson JR. Information Capacity of Discrete Motor Responses. Journal of Experimental Psychology. 1964;67(2):103.

[63] Wu T, Dufford AJ, Mackie MA, Egan LJ, Fan J. The Capacity of Cognitive Control Estimated from a Perceptual Decision Making Task. Scientific Reports. 2016;6(1):34025.

[64] Bialek W, Zee A. Coding and computation with neural spike trains. Journal of Statistical Physics. 1990;59(1):103-115.

[65] Tononi G, Boly M, Massimini M, Koch C. Integrated information theory: from consciousness to its physical substrate. Nature Reviews Neuroscience. 2016;17(7):450461.

[66] Koechlin E, Ody C, Kouneiher F. The architecture of cognitive control in the human prefrontal cortex. Science (New York, NY). 2003;302:1181-5.

[67] Koechlin E, Summerfield C. An information theoretical approach to prefrontal executive function. Trends in Cognitive Sciences. 2007;11(6):229-235. 
[68] Summerfield C, Trittschuh EH, Monti JM, Mesulam MM, Egner T. Neural repetition suppression reflects fulfilled perceptual expectations. Nature neuroscience. $2008 ; 11: 1004-6$.

[69] Alink A, Schwiedrzik CM, Kohler A, Singer W, Muckli L. Stimulus Predictability Reduces Responses in Primary Visual Cortex. Journal of Neuroscience. 2010;30(8):2960-2966.

[70] Todorovic A, van Ede F, Maris E, de Lange FP. Prior expectation mediates neural adaptation to repeated sounds in the auditory cortex: an MEG study. The Journal of neuroscience : the official journal of the Society for Neuroscience. 2011;31:9118-23.

[71] Urgen BA, Miller LE. Towards an Empirically Grounded Predictive Coding Account of Action Understanding. Journal of Neuroscience. 2015;35(12):4789-4791.

[72] Maranesi M, Livi A, Fogassi L, Rizzolatti G, Bonini L. Mirror Neuron Activation Prior to Action Observation in a Predictable Context. Journal of Neuroscience. 2014;34(45):14827-14832.

[73] Kilner JM, Vargas C, Duval S, Blakemore SJ, Sirigu A. Motor activation prior to observation of a predicted movement. Nature neuroscience. 2004;7:1299-301.

[74] Loued-Khenissi L, Preuschoff K. Information Theoretic Characterization of Uncertainty Distinguishes Surprise From Accuracy Signals in the Brain. Frontiers in artificial intelligence. 2020;3:5.

[75] Adams RA, Shipp S, Friston KJ. Predictions not commands: active inference in the motor system. Brain structure \& function. 2013;218:611-43.

[76] Gottwald S, Braun DA. The two kinds of free energy and the Bayesian revolution. PLOS Computational Biology. 2020;16(12):1-32.

[77] Ortega PA, Braun DA. Thermodynamics as a theory of decision-making with information-processing costs. Proceedings of the Royal Society of London A: Mathematical, Physical and Engineering Sciences. 2013;469(2153).

[78] Bhui R, Lai L, Gershman S. Resource-rational decision making. Current Opinion in Behavioral Sciences. 2021;41:15-21.

[79] Tishby N, Polani D. 19. In: Information Theory of Decisions and Actions. New York, NY: Springer New York; 2011. p. 601-636.

[80] Mattsson LG, Weibull JW. Probabilistic choice and procedurally bounded rationality. Games and Economic Behavior. 2002;41(1):61-78.

[81] Friston K. The free-energy principle: a unified brain theory? Nature Reviews Neuroscience. 2010;11(2):127-138.

[82] Berger T. In: Rate Distortion Theory and Data Compression. Vienna: Springer Vienna; 1975. p. 1-39.

[83] Sims CA. Implications of rational inattention. Journal of Monetary Economics. 2003;50(3):665-690.

[84] McKelvey RD, Palfrey TR. Quantal Response Equilibria for Normal Form Games. Games and Economic Behavior. 1995;10(1):6 - 38. 
[85] Sims C, Jacobs R, Knill D. An Ideal Observer Analysis of Visual Working Memory. Psychological review. 2012;119:807-30.

[86] Bhui R, Gershman SJ. Decision by sampling implements efficient coding of psychoeconomic functions. Psychological review. 2018;125:985-1001.

[87] Khodadadi A, Fakhari P, Busemeyer JR. Learning to maximize reward rate: a model based on semi-Markov decision processes. Frontiers in neuroscience. 2014;8:101-101.

[88] Averbeck BB. Theory of Choice in Bandit, Information Sampling and Foraging Tasks. PLOS Computational Biology. 2015;11(3):1-28.

[89] Olshausen BA, Field DJ. Emergence of simple-cell receptive field properties by learning a sparse code for natural images. Nature. 1996;381(6583):607-609.

[90] Olshausen B, Field D. Sparse coding of sensory inputs. Current Opinion in Neurobiology. 2004;14:481-487.

[91] Ortega PA, Braun DA. Information, Utility and Bounded Rationality. In: Schmidhuber J, Thórisson KR, Looks M, editors. Artificial General Intelligence. Berlin, Heidelberg: Springer Berlin Heidelberg; 2011. p. 269-274.

[92] Genewein T, Leibfried F, Grau-Moya J, Braun DA. Bounded Rationality, Abstraction, and Hierarchical Decision-Making: An Information-Theoretic Optimality Principle. Frontiers in Robotics and AI. 2015;2:27. 